\title{
A unique case of borderline lepromatous leprosy presenting with both hypopigmented and hyperpigmented macules
}

\author{
Fazalul Abideen ${ }^{\mathrm{a}}$, Swapna Balakrishnan ${ }^{\mathrm{b}}$, C. B. Sindhu ${ }^{\mathrm{c}}$, \\ Sandhya George ${ }^{\mathrm{d}} \&$ Anita Sanker ${ }^{\mathrm{c}}$ \\ a Junior Consultant in Dermatology, Government Taluk Headquarters \\ Hospital Koilandy, Kerala, India \\ ${ }^{\mathrm{b}}$ Assistant Professor, Department of Pathology, Government Medical \\ College, Manjeri, Kerala, India \\ ${ }^{\mathrm{c}}$ Assistant Professor, Department of Dermatology, Government Medical \\ College, Manjeri, Kerala, India \\ ${ }^{\mathrm{d}}$ Additional Professor, Department of Dermatology, Government Medical \\ College, Manjeri, Kerala, India \\ ORCID: https://orcid.org/0000-0002-5902-2509
}

Submitted 26 May 2021; Accepted 29 October 2021

\begin{abstract}
Summary Primary hyperpigmentation is rare in leprosy. We report a patient with borderline lepromatous (BL) leprosy who presented with both hypopigmented and hyperpigmented macules. Histopathology with Masson Fontana staining and immunohistochemistry with HMB 45 revealed decreased melanin granules in keratinocytes of basal and superficial layers in hypopigmented macules when compared to hyperpigmented macules. It appears that hypopigmentation in leprosy lesions may be due to defective transfer of melanin into keratinocytes.
\end{abstract}

Keywords: Leprosy, hyperpigmented macules, immunohistochemistry, melanocytes, melanin granules

\section{Introduction}

Leprosy, also known as Hansen's disease, is a chronic infectious disease with various epidemiological characteristics and clinical manifestations. ${ }^{1}$ The colour of the lesions in leprosy has been described as hypopigmented, erythematous or coppery coloured. Even though hyperpigmentation secondary to clofazimine or minocycline treatment and, to lepra reaction is common, primary hyperpigmentation in leprosy is a rare feature, ${ }^{2}$ and only a few cases have

Correspondence to: Sandhya George, Additional professor, Department of Dermatology, Government Medical College, Manjeri, Kerala, India (Tel.: 9447570936; e-mail: drsandhyageorge@gmail.com) 
been reported with this. ${ }^{3}$ We report a unique case of borderline lepromatous leprosy presenting with both hypopigmented and hyperpigmented macules.

\section{Case report}

A 20-year-old male painter presented with multiple asymptomatic hypopigmented and hyperpigmented macules over the trunk and extremities, of 5 years duration. Lesions started as asymptomatic small hypopigmented lesions over the trunk and upper limbs, which gradually progressed to larger lesions. A year later multiple asymptomatic hyperpigmented lesions were noticed over both lower limbs, which were also slowly progressive. He gave no history of leprosy in the family or any history of prior drug intake for leprosy. On examination, multiple dry, well to ill-defined hypopigmented macules $(1 \times 1 \mathrm{~cm}$ to $3 \times 4 \mathrm{~cm})$ were noted, over both upper limbs and trunk, with a tendency towards symmetry (Figure 1). The lower limbs showed multiple hypoaesthetic macules of varying sizes $(1 \times 1 \mathrm{~cm}$ to $4 \times 5 \mathrm{~cm})$, with hyperpigmentation in the centre, and a rim of perilesional hypopigmented halos (Figures 2 and 3). On careful examination, the hypopigmented parts were slightly elevated (Figure 2). Sensory examination revealed impairment of pain, touch and temperature sensations in some hypopigmented and some hyperpigmented macules. Neurological examination showed grade 1 thickness bilaterally of the common peroneal, posterior tibial, sural and saphenous nerves. There was no muscle weakness.

Slit skin smears for acid fast bacilli (AFB) were negative from earlobe, lesional and normal skin. Histopathological examination from hypopigmented macules over trunk showed the sub-epidermal Grenz zone with lympho-histiocytic, epithelioid and plasma cell infiltrate in the perineural and periappendage area, especially around the pilo-sebaceous unit (Figures 4 and 5). AFB were absent in Wade Fite stain. The histological diagnosis was mid-borderline leprosy (HD-BB). Two biopsies were taken from lesions on the lower limbs-one from an area of central hyperpigmentation and another from a peripheral hypopigmented area. These biopsies showed similar histopathological findings as in the first biopsy, with a few foamy macrophages also present (Figure 5). Wade Fite staining showed the presence of AFB (Bacterial Index 1-3). This was more suggestive of HDBL. A final diagnosis of HDBL was made based clinical and histopathological features. The patient was started on MB-MDT with a good response to treatment.

To demonstrate the significance of the changes in pigmentation in clinical lesions, we proceeded with special stains, Masson Fontana (Figures 6 and 7), and immuno-histochemistry (IHC) with HMB-45 (Figures 8 and 9) of the sections from hypo and hyperpigmented lesions. We found no significant difference in the melanocyte number in the biopsies from both hyper and hypopigmented areas, but noted a significant decrease in melanin granules in the keratinocytes of both basal and superficial layers in the section from the hypopigmented area, when compared with that from the hyperpigmented area.

\section{Discussion}

Leprosy typically presents with classical features of hypopigmented anaesthetic patches, thickened nerves and the presence of acid-fast bacilli on slit-skin smear examination. However, cases may also present with atypical and uncommon manifestations. Primary hyperpigmentation in leprosy is a rare manifestation, although the secondary pigmentation due to clofazimine or minocycline therapy and lepra reactions is common. ${ }^{2}$ 


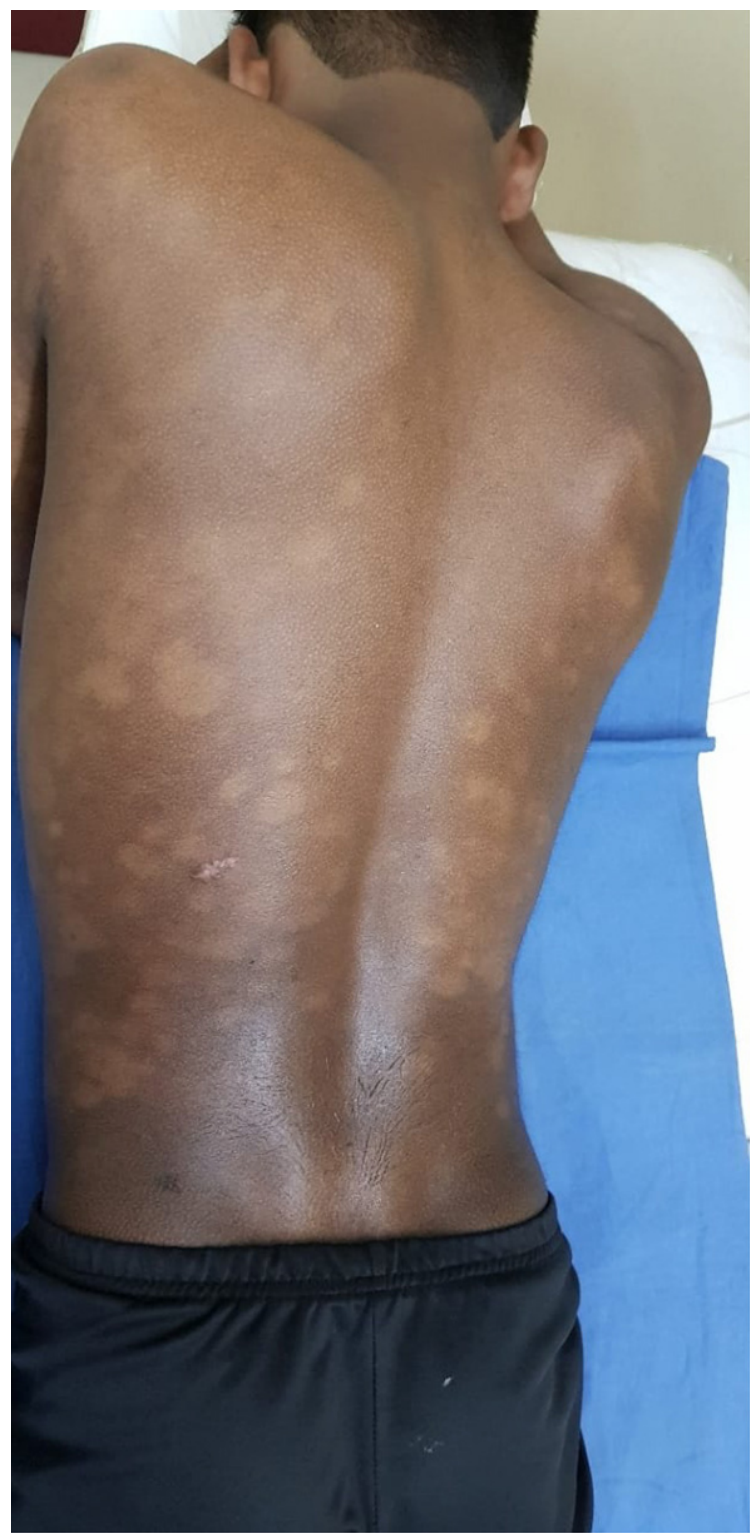

Figure 1. Back of trunk showing multiple hypopigmented macules.

Chattopadhyay et al. reported two such cases, one presenting as a hyperpigmented anaesthetic patch on the pinna of the ear, and the other one as a case of borderline tuberculoid leprosy (HDBT) with Type 1 reaction over the uncommon sites of the palms and soles, with lumbosacral involvement. ${ }^{4}$ Mehta et al. ${ }^{5}$ reported a case of tuberculoid leprosy with hyperpigmented patches on the right leg, while Okhandiar et al. ${ }^{6}$ reported two cases of tuberculoid and HD-BT with hyperpigmented border. Grover et al. ${ }^{7}$ reported a case of HDBT presenting as hyperpigmented patches over the palms. Arakkal et al. ${ }^{2}$ reported HD-BT 


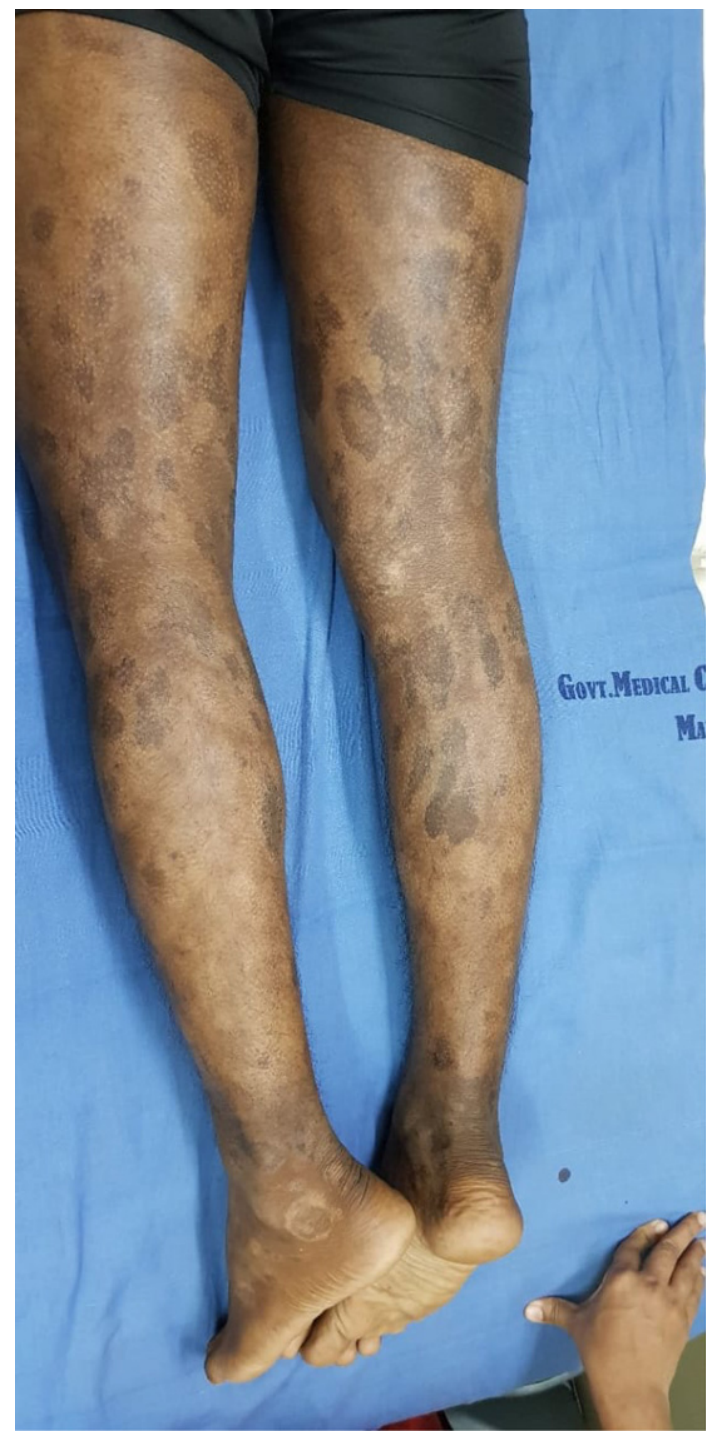

Figure 2. Lower limbs (ventral part) showing multiple hyperpigmented macules with peripheral hypopigmentation. Normal skin colour can be seen in between.

presenting as hyperpigmented patches over the trunk, extremities, palms and soles. Histology revealed increased pigmentation in the basal layer with evidence of BT leprosy. Singh et al. ${ }^{3}$ reported 3 cases of HDBT with a single hyperpigmented lesion over chin, left shin and left ankle, respectively. In one of their cases, the hyperpigmented macule was surrounded by a thin hypopigmented perilesional halo, which has similarity in clinical appearance to our case. Awali et al. ${ }^{8}$ reported a 25 -year-old woman who presented with a hyperpigmented macule on the medial side of her right palm with hypoesthesia over the lesion, diagnosed as HDBT. In the literature, only around 10 cases of leprosy with hyperpigmented macules were reported so far, and all of them were tuberculoid or HDBT. This may be the first case of 


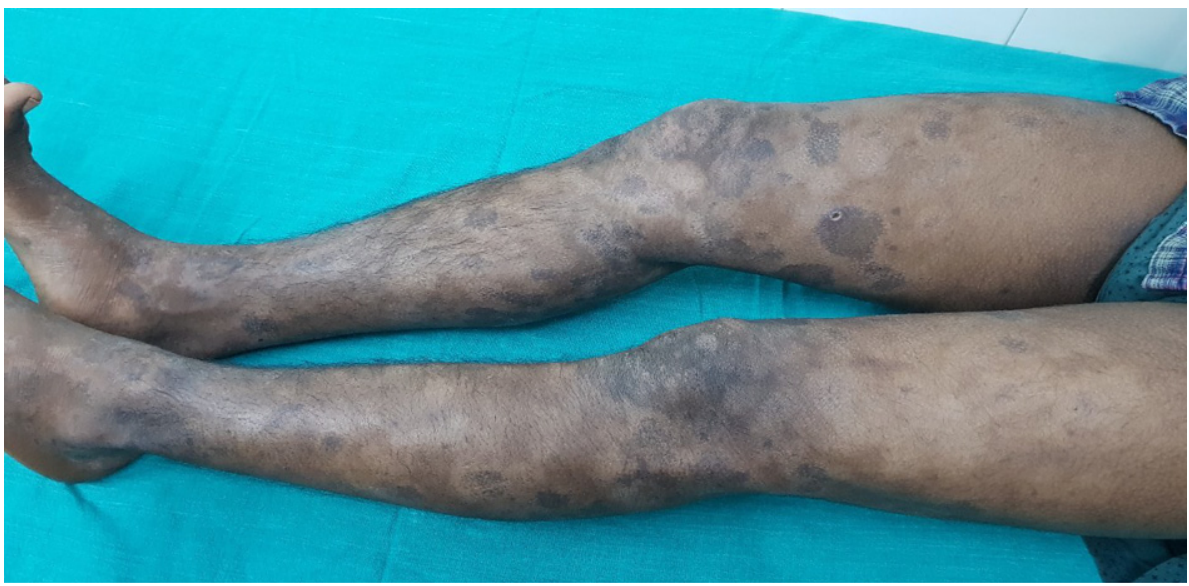

Figure 3. Lower limbs (dorsal part) showing multiple hyperpigmented macules with peripheral hypopigmentation. Normal skin colour can be seen in between.

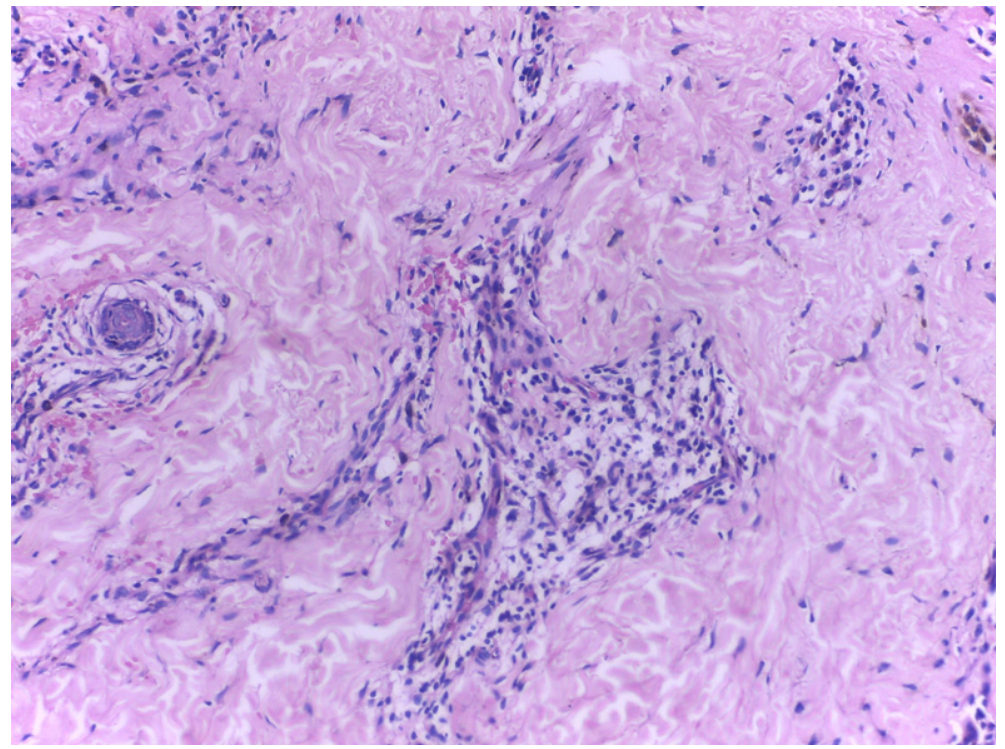

Figure 4. H\&E staining (200x) of skin lesions over trunk, showing vague perineural lympho-histiocytic and epithelioid granulomatous infiltrate.

borderline lepromatous leprosy presenting with hyperpigmented macules with a rim or halo of perilesional hypopigmentation.

The histopathology of HDBB shows an atrophic epidermis, Grenz zone and the presence of AFB. Poorly defined granulomas with epithelioid histiocytes and a few foamy macrophages may also be seen. In HDBL, an atrophic epidermis, Grenz zone, foamy macrophage granulomas and lymphocytes may be seen. ${ }^{9,10}$ Lymphocytes will be more numerous compared to 


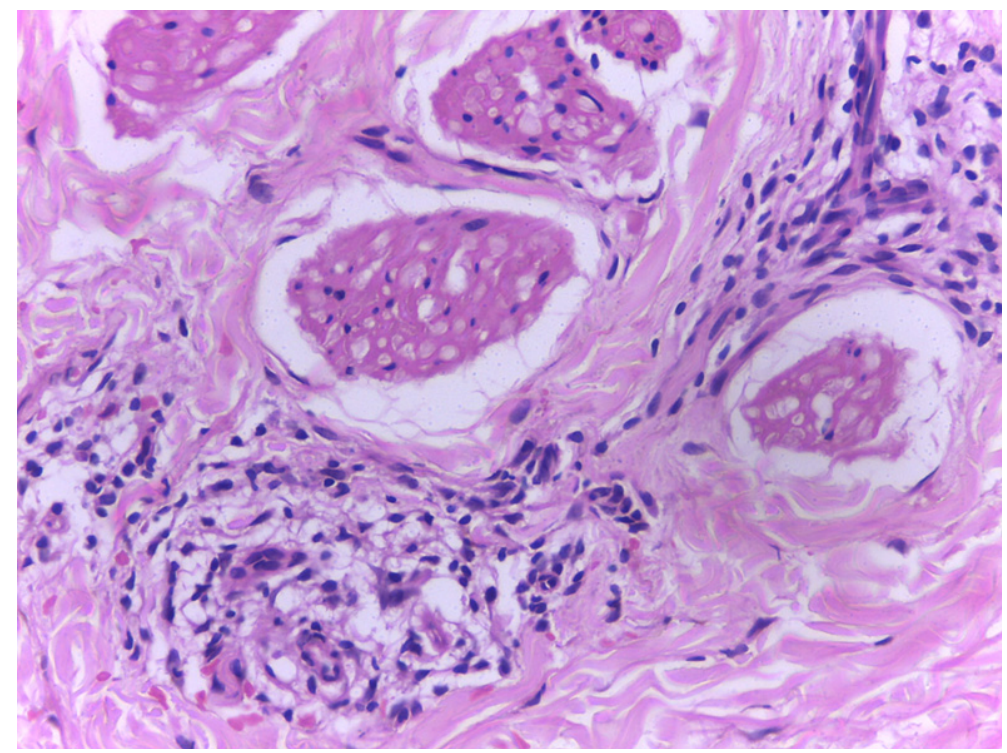

Figure 5. Section of biopsy of skin lesions over lower limb showing perineural infiltrate of foamy histiocytes, lymphocytes, with a few plasma cells and vague onion skin fibrosis. Scanty infiltrate seen around erector pili muscle (Haematoxylin and eosin, 200×).

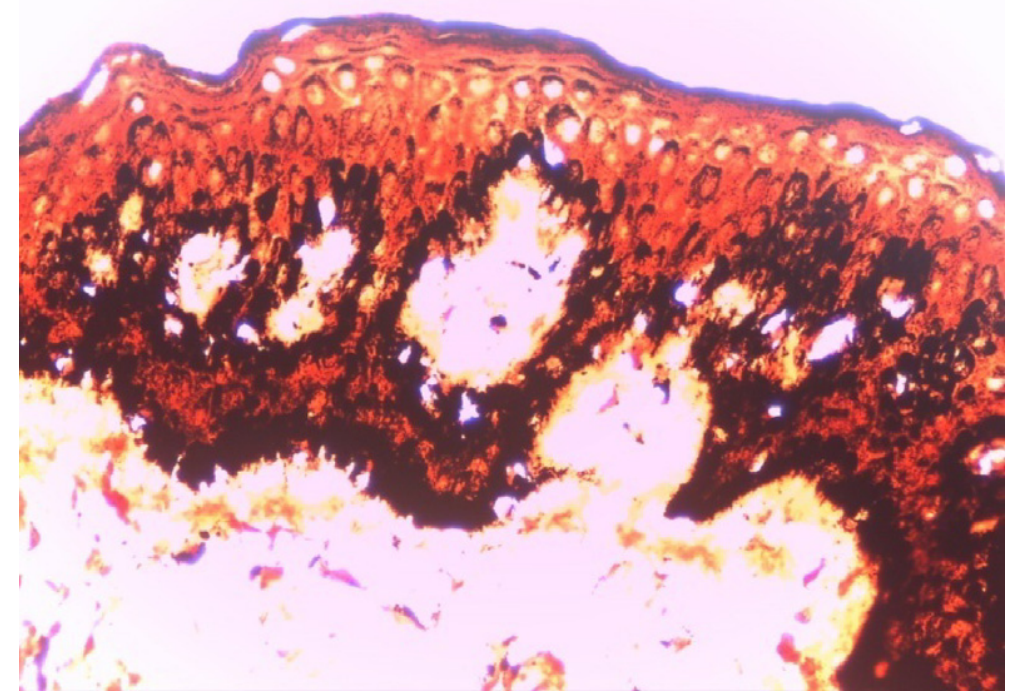

Figure 6. Appearance of melanocytes and superficial keratinocytes with mild decrease of melanin granules in skin biopsy from hypopigmented macules (Masson Fontana stain, 200×).

HDLL. AFB are seen with Wade-Fite stain either singly or forming globi. The histopathology of our case showed features of both HDBB and HDBL. 


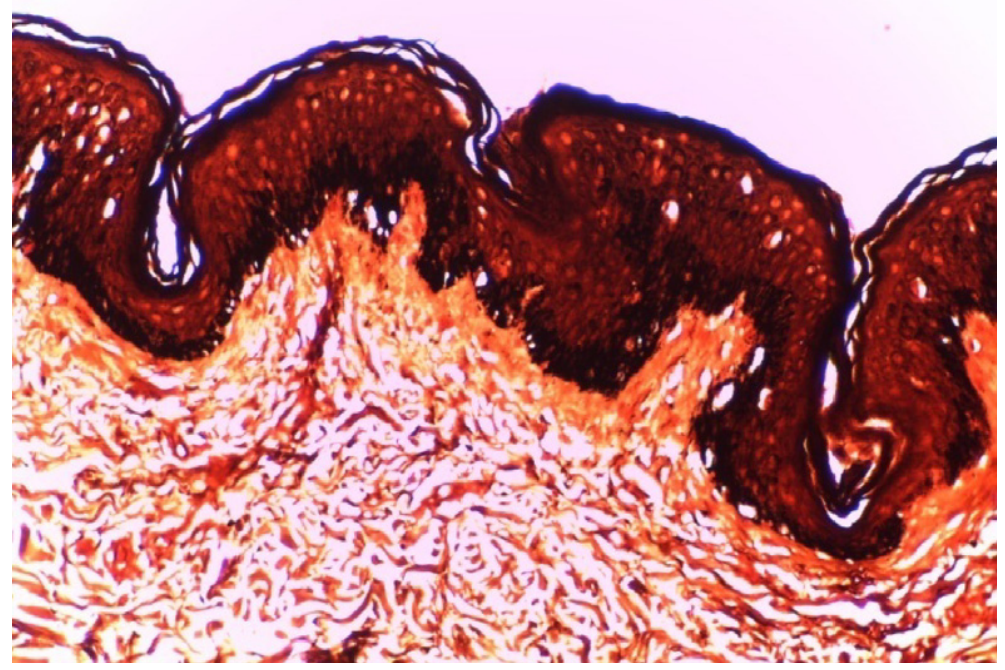

Figure 7. Appearance of melanocytes and superficial keratinocytes with mild increase in melanin granules in skin biopsy from hyperpigmented macules (Masson Fontana stain, 200×).

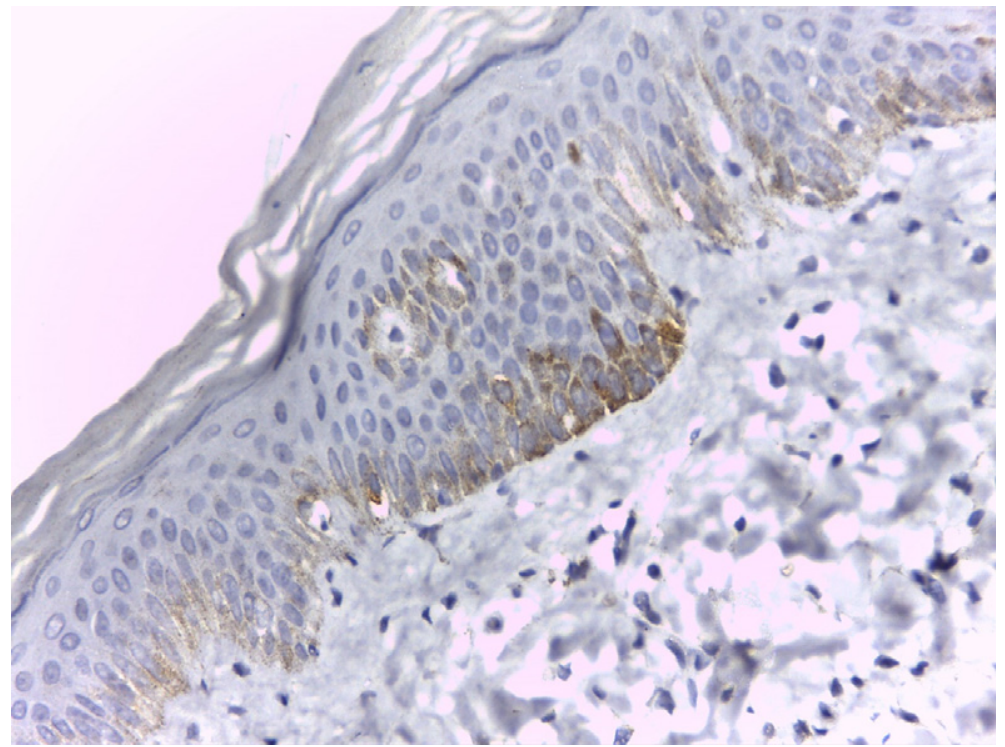

Figure 8. Section of biopsy of skin from hypopigmented area demonstrating appearance of melanocytes with mild decrease in melanin granules (IHC HMB 45, 400×).

A literature review did not reveal any particular reason for hyperpigmentation in leprosy. The hypothesis proposed by Chattopadhyay and Gupta states that hyperpigmentation in leprosy may be due to the over activity of melanocytes, as a result of neuro-hormonal or biochemical 


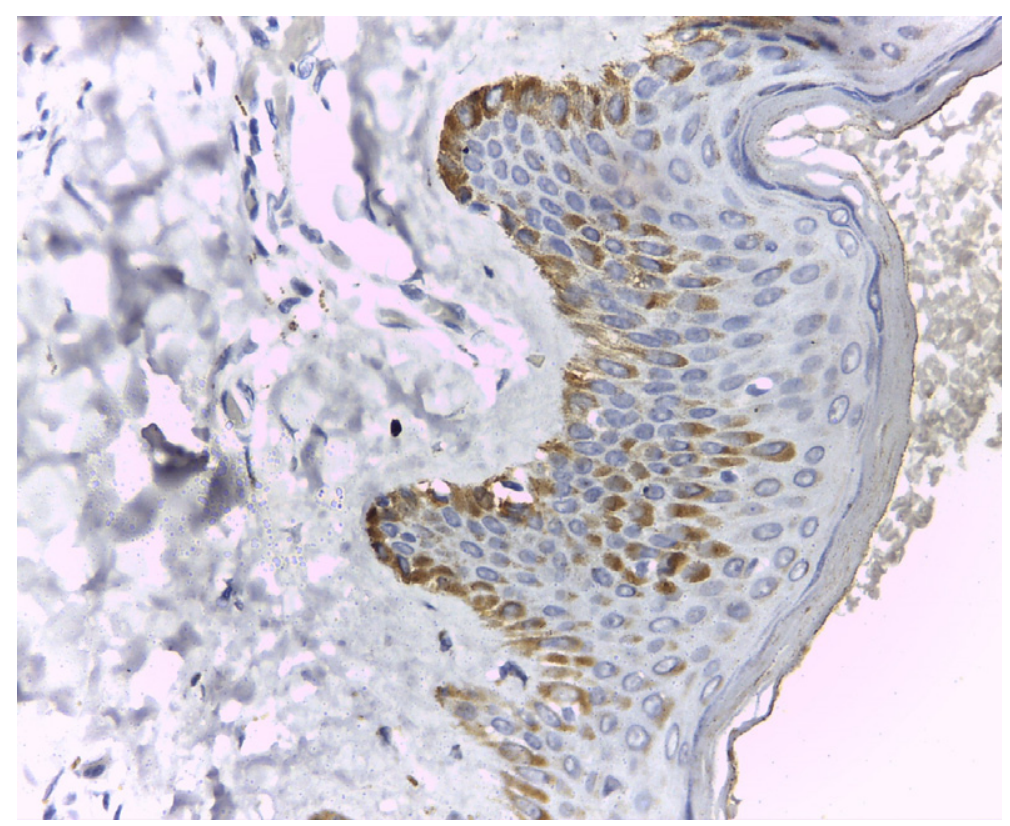

Figure 9. Section of biopsy of skin from hyperpigmented area demonstrating appearance of melanocytes with mild increase in melanin granules (IHC HMB 45, 400x).

stimulation. ${ }^{2}$ Although the exact mechanism of primary hyperpigmentation in leprosy is not clear, they presumed that upregulation of melanogenic stimulating factors like fibroblast growth factor, hepatocyte growth factor and stem cell factors could possibly be responsible. ${ }^{2}$

Shareef et al. ${ }^{11}$ conducted a study in 20 leprosy patients to estimate the number of melanocytes and the amount of pigmentation in hypopigmented lesions and adjacent normal skin, but no differences were found. They postulated that hypopigmentation in leprosy lesions could be caused by defective transfer of melanin into keratinocytes. ${ }^{11}$

In our case, there was a significant reduction in the melanin granules in the keratinocytes of the superficial layers and focally in the basal layer of the epidermis in the biopsies from hypopigmented areas compared to those from hyperpigmented areas. Fontana Masson stain and IHC for HMB 45 demonstrated that the melanocyte number in the basal layers of the epidermis in the biopsies of both areas are not significantly different and the keratinocyte to melanocyte ratio is maintained. Our findings therefore support the hypothesis of a defect in melanocyte-keratinocyte melanosome transfer.

\section{Conclusions}

New, atypical and uncommon presentations of leprosy are still being reported in India during this post-elimination era. We report this case due to its unique presentation, and to suggest that leprosy should be considered in the differential diagnosis of cases of unexplained hyperpigmented lesions. It appears that hypopigmentation in leprosy lesions may be due to defective transfer of melanin into keratinocytes. 


\section{Guarantor}

Dr Sandhya George.

\section{Patient consent}

Patient has given consent to publish his photos.

\section{Conflicts of interest}

No conflict of interest.

\section{Funding}

No funding was paid for this research.

\section{References}

1 Rees RJW, Young DB. The microbiology of leprosy. In: Hastings RC (ed.), Leprosy. 2nd edn, Edinburgh: Churchill Livingstone, 1994.

2 Arakkal GK, Vani S, Kasetty HK, Varala S. Leprosy: An unusual presentation. Int J Med Public Health, 2015; 5: 118-120.

3 Singh SN, Gardin HK, Bhattamishra AB. Hyperpigmented lesions in Hansen's disease. Indian J Dermatol, 1999; 44(4): 215-216.

4 Chattopadhyay SP, Gupta CM. Primary hyperpigmented cutaneous lesions in tuberculoid leprosy. Indian J Lepr, 1988; 60: 63-65.

5 Mehta VR. The primarily pigmented tuberculoid leprosy. Int J Lepr, 1984; 52(Suppl): 687-688

6 Okhandiar RP, Sinha E, Sinha RK. Leprous lesion with hyperpigmented border. Indian J Lepr, 1985; 57: 640643.

7 Grover S, Singh G, Dash K. Primary hyperpigmented palmar lesion: A rare presentation of borderline tuberculoid leprosy. Indian J Lepr, 1997; 69: 191-193.

8 Awali R, Chandrasekar PH. Hyperpigmented macule on the palm and diminished sensation. J Am Med Assoc, 2018; 320(19): 2029-2030. https://doi.org/10.1001/jama.2018.14701.

9 Sasidharanpillai S, Govindan A. Histopathology of leprosy. In: Sardana K, Khurana A (eds), Jopling's Handbook of Leprosy. 6th edn, CBS, 2020.

10 Fischer M. Leprosy-an overview of clinical features, diagnosis, and treatment. J Dtsch Dermatol Ges, 2017; 15(8): 801-827.

11 Shereef PH, Thomas M. Hypopigmented macules in leprosy-a histopathological and histochemical study of melanocytes. Indian J Lepr, 1992; 64(2): 189-191. [published correction appears in Indian J Lepr, 1992; 64(3): 301]. 